\title{
Day and night surgery: is there any influence in the patient postoperative period of urgent colorectal intervention?
}

\author{
Sofia Fernandes ${ }^{2,3}$ - Ana F. Carvalho ${ }^{1,2,3}$ - Ana J. Rodrigues ${ }^{2,3}$ - Patrício Costa ${ }^{2,3}$. \\ Moreno Sanz ${ }^{4}$ - Andre Goulart ${ }^{1}$ - Hugo Rios ${ }^{1}$ • Pedro Leão ${ }^{1,2,3}$
}

Accepted: 22 December 2015 / Published online: 7 January 2016

(C) Springer-Verlag Berlin Heidelberg 2016

\begin{abstract}
Background Medical activity performed outside regular work hours may increase risk for patients and professionals. There is few data with respect to urgent colorectal surgery. The aim of this work was to evaluate the impact of daytime versus nighttime surgery on postoperative period of patients with acute colorectal disease.

Methods A retrospective study was conducted in a sample of patients with acute colorectal disease who underwent urgent surgery at the General Surgery Unit of Braga Hospital, between January 2005 and March 2013. Patients were stratified by operative time of day into a daytime group (surgery between 8:00 and 20:59) and the nighttime group (21:00-7:59) and compared for clinical and surgical parameters. A questionnaire was distributed to surgeons, covering aspects related to the practice of urgent colorectal surgery and fatigue.

Results A total of 330 patients were included, with 214 $(64.8 \%)$ in the daytime group and $116(35.2 \%)$ in the nighttime group. Colorectal cancer was the most frequent pathology. Waiting time $(p<0.001)$ and total length of hospital stay $(p=0.008)$ were significantly longer in the daytime group. There were no significant differences with respect to early or
\end{abstract}

Pedro Leão

pedroleao@ecsaude.uminho.pt

1 General Surgery, Hospital of Braga, Braga, Portugal

2 Life and Health Sciences Research Institute (ICVS), School of Health Sciences, University of Minho, Braga, Portugal

3 ICVS/3B's - PT Government Associate Laboratory, Braga/ Guimarães, Portugal

4 General Surgery, Complejo Hospitalario La Mancha-Centro, Cdad. Real, Spain late complications. However, $100 \%$ of surgeons reported that they are less proficient during nighttime.

Conclusions Among patients with acute colorectal disease subjected to urgent surgery, there was no significant association between nighttime surgery and the presence of postoperative medical and surgical morbidities. Patients who were subjected to daytime surgery had longer length of stay at the hospital.

Keywords After-hours care $\cdot$ Colorectal surgery $\cdot$ Morbidity

\section{Introduction}

According to the report published in 1999 by the Institute of Medicine, medical errors result in more than 98,000 deaths annually in the USA. Surgical complications are considered the second leading preventable cause of morbidity/mortality. Fatigue and excessive working hours are potential factors for medical errors [1]. Medical activity performed outside regular working hours may represent risk for patients and health professionals [2-4]. Doctor's proficiency and situation awareness are smaller at night, and several studies have shown the effects of fatigue and sleep deprivation in performance $[5,6]$. Sleep deprivation affects cognitive and psychomotor performance, leading to a higher risk of surgical complications $[7,8]$.

Several studies have associated nighttime surgery with increased surgical complications, comprising different areas such as organ transplantation, gynecology, orthopedics, etc. $[2-4,9,10]$. Therefore, the most effective method to reduce the complications' risk is rethinking what pathologies and respective presentation constitute a need for nighttime surgery and what interventions should be delayed $[8,11]$. There are several factors involved in this decision: patient factors (comorbidities) that may require optimization before the surgical 
procedure and hospital factors that may arise from elective procedures' list performed during the day, leaving few theaters available to urgent cases [12].

However, other studies show contradictory results. There is no significant association between the operative time of day and survival up to 1 year after thoracic organ transplant [13]. Nighttime kidney transplantation is not associated with increased risk of surgical complications, and survival is independent of the operative time of day [14]. In hip fracture surgery, apart of operative time of day, postoperative complications and postoperative 30-day mortality are comparable, and when an appropriate surgical team is on duty, there was no significant difference between postoperative morbidity and mortality $[15,16]$. Regarding colorectal surgery, it seems that nighttime colorectal surgery is an independent risk factor for anastomotic leakage and patients having nighttime urgent and emergent colorectal surgery were more likely to die $[17,18]$.

The acute colorectal disease can become a life-threatening condition, and opposed to elective procedures, urgent surgery is associated with high rates of morbidity and mortality, even in countries with an excellent health care system [19, 20]. In Portugal, according to the General Direction of Health, 3450 surgeries were performed in colorectal patients with acute complaint, adding up $2.9 \%$ of total urgent surgeries.

Due to controversy and the lack of studies from different hospitals throughout the world about urgent colorectal pathology, we aim to study the influence of nighttime surgery in the postoperative medical and surgical complications.

\section{Patients and methods}

After receiving hospital ethical approval, a retrospective review of medical files was conducted of all patients who underwent urgent colorectal surgery (colorectal pathology with admission on emergency room and patients who required surgical procedure after having been treated on a medical ward) at Hospital Braga institution (teaching hospital) between 1 January 2005 and 31 March 2013, obtaining a total of 330 patients: 197 male patients (59.7\%) and 133 female patients $(40.3 \%)$. Patients with missing data in surgical logbook, undergoing elective or ambulatory surgery, or other procedures out of colorectal segment, or those who had a waiting time to surgery upper than $336 \mathrm{~h}$ were excluded from this study.

The study population was divided into two groups based on the operative start time of surgery to allow comparison of both groups: the daytime group $(n=214,64.8 \%)$-patients who had surgery that started between 8:00 and 20:59 - and the nighttime group $(n=116,35.2 \%)$ - patients who had surgery that started between 21:00 and 7:59. The nighttime period setting is due to the OR nurse shift, since at our institution, they exchange at 8:30/20:30. Medical team has a different urgency shift, performed on $24 \mathrm{~h}$ (8:00-8:00 next day), according to a rotation scheme, including two attending surgeons and two residents. The teams being on call have their own OR during the daytime for urgent procedures. In case of an emergency, the first OR (we have a total of 12 OR) getting empty will be used for the surgical procedure.

Both groups were compared for demographic data, medical co-morbidities (medical pathology history), preoperative status, underlying disease and presentation, site of lesion, waiting time (time between surgical team evaluation and operation time), operative start time, operative details, hospital stay (from admission to discharge), postoperative morbidity (medical and/or surgical complications), and mortality. Patient survival was obtained by calculating the time elapsed between the date of surgery and the date of the last recorded contact, as documented in the medical record. All surgical procedures were supervised by an attending surgeon with at least 10 or more years of practice.

A self-filling, anonymous, and confidential questionnaire was distributed to surgeons of General Surgery Department of Hospital of Braga, covering aspects related to the practice of urgent colorectal surgery and fatigue.

Continuous variables were expressed as means and standard deviations and compared between the two groups using Student's $t$ test. The homogeneity of variance was tested using the Levene test. Categorical variables were expressed as proportions and compared between the two groups using chisquared test or Fisher's exact test. Finally, a multiple linear regression analysis was performed to determine the significant predictor factors of hospital length of stay (postoperative characteristics proxy). With this model, we also have analyzed the effect of operative time of day on dependent variable, knowing the relevant variables.

Data was analyzed in Statistical Package for the Social Sciences version 21.0 (SPSS Inc., Chicago, IL, USA). A $p$ value $<0.05$ was considered significant. Missing values comprise characteristics without applying for specific data or data unavailable for consultation.

\section{Results}

\section{Socio-demographic and clinical-surgical characteristics}

Patients presented a mean age of 66.6 years (range 2-92). Regarding body mass index (BMI), 16 patients $(6.0 \%)$ were underweight and 40 patients $(15.1 \%)$ were obese. Most of the patients $(71.8 \%)$ had no smoking history or alcoholism habits $(65.4 \%)$. About one third of the patients $(38.0 \%)$ had an American Society of Anesthesiologists (ASA) score of II, and a half presented normal levels of C-reactive protein (CRP) (51.5\%). Nearly half of the patients presented normal leucocyte count and hemoglobin level (55.4 and $49.4 \%$, 
respectively). Previous to the surgery, the majority of patients did not present hypoalbuminemia $(80.6 \%)$ or thrombocytosis $(87.9 \%)$ and had no history of steroid use as usual medication $(83.0 \%)$. Almost half of patients $(49.4 \%)$ had prior abdominal surgery. The comparative analysis of the nighttime surgery group and daytime group revealed similar sociodemographic and clinical characteristics (Table 1), except for a high incidence of preoperative anemia in the daytime surgery group $[p=0.008 ; \varphi c=0.207]$.

Regarding co-morbidities, $80.6 \%$ of the patients presented some co-morbidity. Nearly half of the sample (47.9\%) had hypertension. Conversely, the majority of patients displayed no dyslipidemia $(77.8 \%)$, cardiac, or pulmonary comorbidities ( 87.7 and $90.4 \%$, respectively). The comparative analysis found no statistically significant difference.

With respect to the colorectal disease, 136 patients $(41.2 \%)$ had colorectal cancer, the leading disease with urgent indication. The most frequent pathology site was sigmoid location ( $42.6 \%)$. Regarding the clinical presentation, the most common was intestinal obstruction $(44.8 \%)$ followed by peritonitis $(29.7 \%)$. Twenty-two patients presented sepsis $(6.6 \%)$. There were no statistically significant differences between the two groups.

Table 1 Socio-demographic and clinical characteristics

\begin{tabular}{|c|c|c|c|c|c|c|}
\hline Variables & $\begin{array}{l}\text { Total } n=334(100 \%) \\
\text { Mean (SD) }\end{array}$ & $\begin{array}{l}\text { Daytime } n=218(65.3 \%) \\
\text { Mean (SD) }\end{array}$ & $\begin{array}{l}\text { Nighttime } n=116(34.7 \%) \\
\text { Mean (SD) }\end{array}$ & $t(\mathrm{df})$ & $p$ Value & $d$ \\
\hline \multirow[t]{2}{*}{ Age (years) } & $\begin{array}{l}66.55(14.27) \\
0^{\mathrm{b}}\end{array}$ & $67.08(14.05)$ & $65.57(14.67)$ & $0.917(326)$ & 0.360 & 0.13 \\
\hline & $n(\%)$ & $n(\%)$ & $n(\%)$ & $\chi^{2}(d f)$ & $p$ Value & $\phi / \varphi \mathrm{c}$ \\
\hline \multicolumn{7}{|l|}{ Sex } \\
\hline $\begin{array}{l}\text { Male } \\
\text { Female }\end{array}$ & $\begin{array}{l}197(59.7) \\
133(40.3) \\
0^{\mathrm{b}}\end{array}$ & $\begin{array}{l}124(57.9) \\
90(42.1)\end{array}$ & $\begin{array}{l}73(62.9) \\
43(37.1)\end{array}$ & $0.778(1)$ & 0.378 & -0.049 \\
\hline \multicolumn{7}{|l|}{ BMI $\left(\mathrm{kg} / \mathrm{m}^{2}\right)$} \\
\hline $\begin{array}{l}<18.5 \\
18.5-24.9 \\
25-29.9 \\
>30\end{array}$ & $\begin{array}{l}16(6.0) \\
118(44.5) \\
91(34.1) \\
40(15.1) \\
67^{\mathrm{b}}\end{array}$ & $\begin{array}{l}11(6.4) \\
85(49.4) \\
50(29.1) \\
26(15.1)\end{array}$ & $\begin{array}{l}5(5.4) \\
33(35.5) \\
41(44.1) \\
14(15.1)\end{array}$ & $6.700(3)$ & 0.082 & 0.159 \\
\hline \multicolumn{7}{|l|}{ ASA score } \\
\hline $\begin{array}{l}\text { I } \\
\text { II } \\
\text { III } \\
\text { IV } \\
\text { V }\end{array}$ & $\begin{array}{l}37(11.2) \\
125(38.0) \\
103(32.9) \\
58(17.6) \\
6(1.8) \\
1^{b}\end{array}$ & $\begin{array}{l}19(8.9) \\
80(37.6) \\
70(32.9) \\
40(18.8) \\
4(1.9)\end{array}$ & $\begin{array}{l}18(15.5) \\
45(38.8) \\
33(28.4) \\
18(15.5) \\
2(1.7)\end{array}$ & & $0.439^{\mathrm{a}}$ & 0.108 \\
\hline \multicolumn{7}{|c|}{ WBC count (cells/ml) } \\
\hline $\begin{array}{l}\text { Normal } \\
\geq 10.000\end{array}$ & $\begin{array}{l}175(55.4) \\
139(44.6) \\
16^{\mathrm{b}}\end{array}$ & $\begin{array}{l}120(59.1) \\
83(40.9)\end{array}$ & $\begin{array}{l}54(48.6) \\
57(51.4)\end{array}$ & & $0.149^{\mathrm{a}}$ & 0.150 \\
\hline \multicolumn{7}{|c|}{ Hemoglobin levels (g/dl) } \\
\hline $\begin{array}{l}\text { Normal } \\
0(\geq 11.0) \\
\text { I }(9.5-10.9) \\
\text { II }(8.0-9.4) \\
\text { III }(6.5-7.9) \\
\text { IV }(<6.5)\end{array}$ & $\begin{array}{l}155(49.4) \\
55(17.5) \\
58(18.5) \\
32(10.2) \\
10(3.2) \\
4(1.3) \\
16^{\mathrm{b}}\end{array}$ & $\begin{array}{l}89(43.8) \\
36(17.7) \\
39(19.2) \\
29(14.3) \\
7(3.4) \\
3(1.5)\end{array}$ & $\begin{array}{l}66(59.5) \\
19(17.1) \\
19(17.1) \\
3(2.7) \\
3(2.7) \\
1(0.9)\end{array}$ & & $0.008^{\mathrm{a}}$ & 0.207 \\
\hline
\end{tabular}

The significant $p$ values are in bold

$n$ absolute frequency, \% relative frequency, $S D$ standard deviation, $t$ Student's $t$ test, $\chi^{2}$ chi-squared test, $d f$ degrees of freedom, $d$ Cohen's $d, \phi$ Pearson's Phi correlation coefficient, $\varphi c$ Cramer's V, ASA American Society of Anesthesiologists

${ }^{\mathrm{a}}$ Fisher exact test

${ }^{\mathrm{b}}$ Missing values 
The mean waiting time was $40.86 \mathrm{~h}$ (range 1-315 h). Furthermore, the mean operative time was $107.11 \mathrm{~min}$ (range 12-315 min). The leading surgeon was most of the time an attending surgeon $(68.6 \%)$.

With respect to the procedure, Hartmann's procedure $(27.2 \%)$ was the most selected, followed by stoma procedure $(25.7 \%)$. Of the procedures, $15.2 \%$ were palliative versus curative intent. Almost all of the cases presented no intraoperative complications (96.7\%) or iatrogenic injuries $(91.8 \%$ ) nor needed blood transfusion (76.4\%).

No statistically significant difference was found between the groups with respect to colorectal disease and surgical characteristics (Table 2) except for patients of daytime group who had a longer waiting time than the nighttime group $[t(236)=5.96 ; p<0.001 ; d=0.76]$.

\section{Postoperative morbidity analysis}

Analysis of postoperative characteristics is summarized in Table 3. The mean hospital length of stay was 18 days (range 1-159), and the mean intensive care unit (ICU) length of stay after surgery was 2 days (range 0-89). Moreover, the majority of patients were not hospitalized in ICU (77.2\%) after surgery nor had necessity of relaparotomy ( $86.5 \%)$. Most of the patients $(61.5 \%)$ had no postoperative medical morbidity, and less than a half had surgical morbidity (32.7\%). With respect to ClavienDindo classification [21], $29.9 \%$ of the patients presented grade 0 , followed by grade $5(25.0 \%)$ and grade $2(18.0 \%)$. The statistical analysis demonstrates differences between groups regarding length of stay $[t(286)=2.672 ; p=0.008 ; d=0.322]$, with increased mean length of stay in daytime group.

With respect to early complications, $4.9 \%$ of the patients had wound infection, $3.4 \%$ had stoma complication, $4.0 \%$ had anastomostic dehiscence and $5.8 \%$ dehiscence of the suture, $9.8 \%$ had abscess, $6.1 \%$ had enterocutaneous fistula, and $2.2 \%$ had colorectal bleeding. Overall, the mortality rate was $25.0 \%$ at postoperative 30 days.

Between the surgery and the last contact with hospital, a total mean of 24 months elapsed (range 0-107). About $13.1 \%$ of patients had incisional hernia, $39.2 \%$ had a definitive stoma, and $14.9 \%$ had a relapse of the colorectal disease. Overall, the mortality rate at postoperative 6 months was $34.1 \%$. There was no difference statistically significant between the groups.

\section{Multiple linear regression model predictor of hospital length of stay}

The multiple regression model with the considered set of predictors was found to be significant $[F(9,220)=33.016$; $\left.p<0.001 ; R_{a}^{2}=0.557\right]$ (Table 4). The significant predictors were ICU length of stay $(\beta=0.525 ; t(220)=11.283$; $p<0.001)$, waiting time $(\beta=0.301 ; t(220)=6.417$; $p<0.001)$, relaparotomy $(\beta=0.277 ; t(220)=5.902$; $p<0.001)$, morbidity $(\beta=0.103 ; t(220)=2.13 ; p<0.028)$, and co-morbidity $(\beta=0.099 ; t(220)=2.103 ; p=0.011)$. Despite statistically significant differences between the two groups with respect to length of stay (Table 3), the operative time of day was not a significant predictor of length of stay in this model. Nevertheless, this model explains about half of the variability in the length of hospitalization. In other words, $55.7 \%$ of the variability in the hospital length of stay can be explained by the significant predictors named above.

\section{Surgeon survey}

Surgeons reported (Table 5) that they have worked in the week preceding the shift period, about $53.70 \mathrm{~h}$ and they slept a mean of $5.8 \mathrm{~h}$ (range 3.5-8.0) in the preceding $24 \mathrm{~h}$. Of the surgeons, $57.1 \%$ rated their fatigue level during nighttime (urgency shift) as "very tired," and $100 \%$ considered less able to operate in nighttime. At least, $59.1 \%$ agreed to postpone surgery safely.

\section{Discussion}

Despite all the studies done in colorectal surgery [17, 18], the present survey is the only analysis evaluating the relationship between nighttime urgent colorectal surgery and medical and surgical morbidity, a particularly important issue, especially concerning the patient safety.

With respect to clinical findings in preoperative time, patients operated during daytime present higher anemia rate. However, this is only a clinical finding, since using multivariate analysis, anemia does not predict a longer hospital length of stay nor there are other statistically significant associations that could be explained by this finding.

Regarding the waiting time, patients from daytime group waited longer to be operated. Patients admitted during daytime were probably postponed until the day after presentation or a day or two after that, since they did not constitute indication to surgical procedure (for example, in diverticulitis, we try first the conservative treatment, only in the case of failure we do the intervention). Another reason might be the availability of $\mathrm{OR}$ for nighttime period after scheduled cases have been completed and also the decision of the surgeon to postpone surgery for next day, since $100 \%$ of surgeons considered themselves less able to operate in nighttime. At least, $59.1 \%$ agreed, to postpone surgery safely for the next morning by several factors: patients (lack of surgical conditions, anticoagulation), logistics (availability of $\mathrm{OR}$ ), and surgical team (fatigue). Fatigue is a crucial factor as having a substantial role in medical errors [1]. Indeed, physicians with nighttime professional activity have their cognitive and psychomotor performance committed $[22,23]$. Of the surgeons, $57.1 \%$ rated their fatigue level during nighttime (last urgency shift) as very tired. This is due to the fact that in the preceding $24 \mathrm{~h}$, they slept a mean of $5.8 \mathrm{~h}$ (range 3.5-8.0) and 
Table 2 Colorectal disease and surgical characteristics

\begin{tabular}{|c|c|c|c|c|c|c|}
\hline Variables & $\begin{array}{l}\text { Total } n=334(100 \%) \\
\text { Mean (SD) }\end{array}$ & $\begin{array}{l}\text { Daytime } n=218(65.3 \%) \\
\text { Mean (SD) }\end{array}$ & $\begin{array}{l}\text { Nighttime } n=116(34.7 \%) \\
\text { Mean (SD) }\end{array}$ & $t(\mathrm{df})$ & $p$ Value & $d$ \\
\hline \multirow[t]{2}{*}{ Waiting time $(\mathrm{h})$} & $\begin{array}{l}40.86(64.19) \\
91^{\mathrm{b}}\end{array}$ & $57.55(74.81)$ & $16.00(30.25)$ & $5.96(237)$ & $<0.001$ & 0.76 \\
\hline & $n(\%)$ & $n(\%)$ & $n(\%)$ & $\chi^{2}(\mathrm{df})$ & $p$ Value & $\phi / \varphi \mathrm{c}$ \\
\hline \multicolumn{7}{|l|}{ Colorectal disease } \\
\hline Colorectal cancer & $136(41.2)$ & $92(43.0)$ & $44(37.9)$ & & $0.157^{\mathrm{a}}$ & 0.177 \\
\hline Anastomotic dehiscence & $56(17.0)$ & $39(18.2)$ & $17(14.7)$ & & & \\
\hline Diverticulitis & $37(11.2)$ & $17(7.9)$ & $20(17.2)$ & & & \\
\hline Iatrogenic perfuration & $19(5.8)$ & $12(5.6)$ & $7(6.0)$ & & & \\
\hline Volvulus & $16(5.0)$ & $8(3.7)$ & $8(6.9)$ & & & \\
\hline Vascular disease & $13(3.9)$ & $8(3.7)$ & $5(4.3)$ & & & \\
\hline Inflammatory bowel disease & $2(0.6)$ & $2(0.9)$ & $0(0)$ & & & \\
\hline \multirow[t]{2}{*}{ Other } & $51(15.5)$ & $36(16.8)$ & $15(12.9)$ & & & \\
\hline & $12^{\mathrm{b}}$ & & & & & \\
\hline \multicolumn{7}{|l|}{ Presentation } \\
\hline Peritonitis & $98(29.7)$ & $57(26.6)$ & $41(35.3)$ & & $0.175^{\mathrm{a}}$ & 0.157 \\
\hline Sepsis & $22(6.7)$ & $18(8.4)$ & $4(3.4)$ & & & \\
\hline Obstruction & $148(44.8)$ & $98(45.8)$ & $50(43.1)$ & & & \\
\hline Hemorrhage & $10(3.0)$ & $9(4.2)$ & $1(0.9)$ & & & \\
\hline Perforation & $22(6.7)$ & $13(6.1)$ & $9(7.8)$ & & & \\
\hline Abscess & $7(2.1)$ & $4(1.9)$ & $3(2.6)$ & & & \\
\hline \multirow[t]{2}{*}{ Other } & $23(7.0)$ & $15(7.0)$ & $8(6.9)$ & & & \\
\hline & $0^{\mathrm{b}}$ & & & & & \\
\hline \multicolumn{7}{|l|}{ Qualification } \\
\hline Resident & $100(30.6)$ & $65(30.5)$ & $35(30.7)$ & $0.010(1)$ & 0.972 & 0.02 \\
\hline \multirow[t]{2}{*}{ Attending surgeon } & $227(68.6)$ & $148(69.5)$ & $79(69.3)$ & & & \\
\hline & $3^{\mathrm{b}}$ & & & & & \\
\hline \multicolumn{7}{|l|}{ Procedure } \\
\hline Right (extended) hemicolectomy & $54(16.5)$ & $38(18.0)$ & $16(13.8)$ & & $0.355^{\mathrm{a}}$ & 0.164 \\
\hline Left hemicolectomy & $8(2.4)$ & $7(3.3)$ & $1(0.9)$ & & & \\
\hline Sigmoidectomy & $15(4.6)$ & $10(4.7)$ & $5(4.3)$ & & & \\
\hline Segmentar colectomy & $15(4.6)$ & $8(3.8)$ & $7(6.0)$ & & & \\
\hline Subtotal colectomy & $17(5.2)$ & $12(5.7)$ & $5(4.3)$ & & & \\
\hline Total colectomy & $1(0.3)$ & $1(0.5)$ & $0(0)$ & & & \\
\hline Hartmann's procedure & $89(27.2)$ & $52(24.6)$ & $37(31.9)$ & & & \\
\hline Stoma procedure & $84(25.7)$ & $53(25.1)$ & $31(26.7)$ & & & \\
\hline Anterior resection & $5(1.5)$ & $5(2.4)$ & $0(0)$ & & & \\
\hline Bowel suture & $15(4.6)$ & $9(4.3)$ & $6(5.2)$ & & & \\
\hline \multirow[t]{2}{*}{ Other } & $24(7.3)$ & $16(7.6)$ & $8(6.9)$ & & & \\
\hline & $4^{\mathrm{b}}$ & & & & & \\
\hline \multicolumn{7}{|l|}{ Intraoperative complication } \\
\hline Yes & $11(3.3)$ & & $7(3.3)$ & $4(3.4)$ & $1.000^{\mathrm{a}}$ & 0.005 \\
\hline \multirow[t]{2}{*}{ No } & $319(96.7)$ & & $207(96.7)$ & $112(96.6)$ & & \\
\hline & $0^{\mathrm{b}}$ & & & & & \\
\hline
\end{tabular}

The significant $p$ values are in bold

$n$ absolute frequency, \% relative frequency, $S D$ standard deviation, $t$ Student's $t$ test, $\chi^{2}$ chi-squared test, $d f$ degrees of freedom, $d$ Cohen's $d, \phi$ Pearson's Phi correlation coefficient, $\varphi c$ Cramer's $V$

${ }^{\mathrm{a}}$ Fisher exact test

${ }^{\mathrm{b}}$ Missing values 
Table 3 Postoperative characteristics

\begin{tabular}{|c|c|c|c|c|c|c|}
\hline Variables & $\begin{array}{l}\text { Total } n=334(100 \%) \\
\text { Mean (SD) }\end{array}$ & $\begin{array}{l}\text { Daytime } n=218(65.3 \%) \\
\text { Mean (SD) }\end{array}$ & $\begin{array}{l}\text { Nighttime } n=116(34.7 \%) \\
\text { Mean (SD) }\end{array}$ & $t(\mathrm{df})$ & $p$ Value & $d$ \\
\hline \multirow[t]{2}{*}{ Hospital length of stay (days) } & $\begin{array}{l}18.74(21.09) \\
5^{\mathrm{b}}\end{array}$ & $20.87(22.58)$ & $14.84(17.49)$ & $2.672(287)$ & 0.008 & 0.322 \\
\hline & $n(\%)$ & $n(\%)$ & $n(\%)$ & $\chi^{2}(\mathrm{df})$ & $p$ Value & $\phi$ \\
\hline \multicolumn{7}{|l|}{ ICU hospitalization } \\
\hline Yes & $75(22.8)$ & $51(23.9)$ & $24(20.7)$ & $0.452(1)$ & 0.501 & -0.037 \\
\hline No & $\begin{array}{l}254(77.2) \\
1^{\mathrm{b}}\end{array}$ & $162(76.1)$ & $92(79.3)$ & & & \\
\hline \multicolumn{7}{|l|}{ Relaparotomy } \\
\hline Yes & $44(13.5)$ & $31(14.7)$ & $13(11.4)$ & $0.684(1)$ & 0.408 & -0.046 \\
\hline No & $\begin{array}{l}281(86.5) \\
5^{\mathrm{b}}\end{array}$ & $180(85.3)$ & $101(88.6)$ & & & \\
\hline \multicolumn{7}{|l|}{ Medical morbidity } \\
\hline Yes & $102(38.5)$ & $68(40.7)$ & 34 (34.7) & $0.947(1)$ & 0.362 & -0.060 \\
\hline No & $\begin{array}{l}163(61.5) \\
\mathbf{6 6}^{\mathrm{b}}\end{array}$ & $99(59.3)$ & $64(65.3)$ & & & \\
\hline \multicolumn{7}{|l|}{ Surgical morbidity } \\
\hline $\begin{array}{l}\text { Yes } \\
\text { No }\end{array}$ & $\begin{array}{l}107(32.7) \\
220(67.3) \\
3^{b}\end{array}$ & $\begin{array}{l}75(35.4) \\
137(64.6)\end{array}$ & $\begin{array}{l}32(27.8) \\
83(72.2)\end{array}$ & $1.931(1)$ & 0.165 & -0.077 \\
\hline \multicolumn{7}{|l|}{ Wound infection } \\
\hline $\begin{array}{l}\text { Yes } \\
\text { No }\end{array}$ & $\begin{array}{l}16(4.9) \\
311(94.9) \\
3^{b}\end{array}$ & $\begin{array}{l}10(4.7) \\
202(95.3)\end{array}$ & $\begin{array}{l}6(5.2) \\
109(94.8)\end{array}$ & $0.04(1)$ & 0.841 & 0.011 \\
\hline \multicolumn{7}{|l|}{ Anastomostic dehiscence } \\
\hline $\begin{array}{l}\text { Yes } \\
\text { No }\end{array}$ & $\begin{array}{l}13(4.0) \\
315(96.0) \\
2^{\mathrm{b}}\end{array}$ & $\begin{array}{l}9(4.2) \\
203(95.8)\end{array}$ & $\begin{array}{l}4(3.4) \\
112(96.6)\end{array}$ & & $0.488^{\mathrm{a}}$ & -0.020 \\
\hline \multicolumn{7}{|l|}{ Abscess } \\
\hline $\begin{array}{l}\text { Yes } \\
\text { No }\end{array}$ & $\begin{array}{l}32(9.8) \\
295(90.2) \\
3^{b}\end{array}$ & $\begin{array}{l}21(9.9) \\
191(90.1)\end{array}$ & $\begin{array}{l}11(9.6) \\
104(90.4)\end{array}$ & $0.010(1)$ & 0.921 & -0.005 \\
\hline \multicolumn{7}{|l|}{ Clavien-Dindo } \\
\hline 0 & $98(29.9)$ & $56(26.3)$ & $42(36.5)$ & & & \\
\hline 1 & $29(8.8)$ & $20(9.4)$ & $9(7.8)$ & & & \\
\hline 2 & $59(18.0)$ & $42(19.7)$ & $17(14.8)$ & & & \\
\hline $3 \mathrm{a}^{\mathrm{a}}$ & $0(0)$ & $0(0)$ & $0(0)$ & & & \\
\hline $3 b$ & $33(10.1)$ & $22(10.3)$ & $11(9.6)$ & & $0.303^{\mathrm{a}}$ & 0.120 \\
\hline $4 a^{a}$ & $14(4.3)$ & $9(4.2)$ & $5(4.3)$ & & & \\
\hline $4 \mathrm{~b}$ & $13(4.0)$ & $10(4.7)$ & $3(2.6)$ & & & \\
\hline 5 & $\begin{array}{l}82(25.0) \\
2^{\mathrm{b}}\end{array}$ & $54(25.4)$ & $28(24.3)$ & & & \\
\hline \multicolumn{7}{|l|}{ 30-day mortality } \\
\hline Yes & $82(25.0)$ & $54(25.4)$ & $28(24.3)$ & $0.040(1)$ & 0.894 & -0.011 \\
\hline No & $\begin{array}{l}246(75.0) \\
2^{b}\end{array}$ & 159 (74.6) & $87(75.7)$ & & & \\
\hline
\end{tabular}

The significant $p$ values are in bold

$n$ absolute frequency, \% relative frequency, $S D$ standard deviation, $t$ Student's $t$ test, $\chi^{2}$ chi-squared test, $d f$ degrees of freedom, $d$ Cohen's $d, \phi$ Pearson's Phi correlation coefficient, $\varphi c$ Cramer's $V$

${ }^{\mathrm{a}}$ Fisher exact test

${ }^{\mathrm{b}}$ Missing values 
Table 4 Model of multiple linear regression predictor the variable hospital length of stay

\begin{tabular}{lrlllll}
\hline Variables & $B$ & SE & $\beta$ & $t$ & $p$ Value & CI 95\% \\
\hline Constant & 4.646 & 3.446 & & 1.348 & 0.179 & {$[-2.146 ; 11.438]$} \\
Age & -0.012 & 0.050 & -0.011 & -0.241 & 0.809 & {$[-0.111 ; 0.087]$} \\
Co-morbidity & 3.975 & 1.890 & 0.099 & 2.103 & $\mathbf{0 . 0 3} *$ & {$[0.249 ; 7.700]$} \\
Hypoalbuminemia & 3.699 & 2.034 & 0.082 & 1.818 & 0.070 & {$[-0.310 ; 7.707]$} \\
Operative time of day & -1.512 & 1.509 & -0.047 & -1.002 & 0.318 & {$[-4.486 ; 1.463]$} \\
Intraoperative complications & -3.585 & 3.882 & -0.041 & -0.923 & 0.357 & {$[-11.235 ; 4.066]$} \\
Relaparotomy & 13.427 & 2.275 & 0.277 & 5.902 & $\mathbf{0 . 0 0 0} * *$ & {$[8.944 ; 17.911]$} \\
Morbidity & 3.454 & 1.561 & 0.103 & 2.213 & $\mathbf{0 . 0 2 8}$ & {$[0.378 ; 6.529]$} \\
ICU length of stay & 1.167 & 0.103 & 0.525 & 11.283 & $\mathbf{0 . 0 0 0 * *}$ & {$[0.964 ; 1.371]$} \\
Waiting time & 0.051 & 0.008 & 0.301 & 6417 & $\mathbf{0 . 0 0 0}$ & {$[0.036 ; 0.067]$} \\
\hline
\end{tabular}

$R^{2}=0.575 ; R_{a}^{2}=0.557 ; p<0.001 ; 0$ - daytime surgery; 1 - nighttime surgery. The significant $p$ values are in bold

$B$ nonstandard regression coefficient, $S E$ standard error, $\beta$ standard regression coefficient, $t$ test value, $C I$ confidence interval

$* p<0.05 ; * * p<0.001$

healthy adults require a sleep daily average of $8 \mathrm{~h}$ [24]. Partial sleep deprivation $(<5 \mathrm{~h})$ has an impact on clinical proficiency [25]. To prevent medical errors, there are restrictions regarding working hours. At our institution, urgency shifts function according to a rotation scheme in which each team performs a

Table 5 Surgeon questionnaire data

\begin{tabular}{ll}
\hline & Total $n=22(100 \%)$ \\
\hline & Mean (SD) \\
Age (years) & $39.23(13.58)$ \\
Sleep hours $/ 24 \mathrm{~h}^{\mathrm{a}}(\mathrm{h})$ & $5.8(1.8)$ \\
Work (hours preceding week) & $\mathrm{a}$ \\
& $53.70(21.30)$ \\
Qualification & $n(\%)$ \\
Attending surgeon & \\
Resident & $11(50)$ \\
Colorectal surgery experience & $11(50)$ \\
Yes & \\
No & $21(95.5)$ \\
Period of lesser ability & $1(4.5)$ \\
Nighttime & \\
Daytime & $21(100)$ \\
Postpone urgent surgery? & $0(0)$ \\
Yes & \\
No & $7(35)$ \\
Fatigue level (nighttime) & \\
None & $13(65)$ \\
Little tired & \\
Tired & \\
Very tired & $0(0)$ \\
Exhausted & $1(4.8)$ \\
\hline & $6(28.6)$ \\
& $12(57.1)$ \\
\hline &
\end{tabular}

${ }^{\text {a }}$ Last urgency shift 24-h shift per week. Surgeons reported that they have worked in the week preceding the shift period, about $53.70 \mathrm{~h}$, higher than legislation stipulated [26].

Nevertheless, no statistically significant differences were found between the medical and surgical morbidity, which contrasts with the existing literature, which showed higher rate of anastomotic leakage in patients undergoing nighttime colorectal surgery and increased mortality in patients undergoing nighttime urgent/emergency surgery in relation to daytime surgery [17, 18]. Unlike Elshove-Bolk et al. [18] that demonstrated less involvement of attending surgeons in nighttime colorectal surgery, this study showed that they performed the leading role in most of the nighttime surgeries. This is particularly relevant in the light of evidence suggesting that patients undergoing colorectal urgent surgery by an unsupervised resident are at increased risk of postoperative complications [27].

Daytime group had significantly greater overall length of stay, unlike shown by Lee et al. [2]. Despite that, there were no differences in co-morbidities and postoperative morbidity or mortality between both groups. Perhaps daytime surgery patients were truly more urgent and therefore sicker than were the nighttime patients, or the longer delay before daytime surgery allowed a greater progression of the acute colorectal disease, which could lead to a longer postoperative recovery.

Using a multiple linear regression model, length of stay predicting factors are waiting time, ICU length of stay, since they are included in hospital length of stay; relaparotomy and postoperative morbidity (medical and/or surgical), since they add more days to the postoperative period in order to restore functions or to fix a problem associated with postoperative complications. Surprisingly, operative time of day did not seem to predict significantly hospital length of stay, and patients subjected to nighttime surgery presented with significantly shorter hospital length of stay. 
With respect to overall mortality rate, several studies report a rate between 6.2 and $28.3 \%$ [27-29]. However, only one study about acute disease included several etiologies and presentations, which presented a mortality rate of $16.4 \%$ and a morbidity rate of $53.4 \%$ [25]. The present study demonstrated an overall mortality rate at 30 days about $25.0 \%$. This higher rate can be explained by the fact that this study included several etiologies and acute pathology with urgent surgical indication, in which it is not possible to optimize patient's clinical condition.

Despite the interesting results, there are inherent limitations to this study. A retrospective study in a single center with a nonrandomized sample selected by convenience can create a selection bias. However, the sample size has sufficient statistical power which allowed us to establish significant associations. Regarding the compiled data, there is also a bias, since data were collected from medical files, which is operator-dependent.

Nevertheless, we believe that more studies are needed to understand the relation between colorectal urgency surgery and the operatory time of day, as for instance a study that would evaluate the survival in a long- and short-term basis. The best option would be to perform a prospective multicenter study. Furthermore, it is necessary to understand which factors are associated with this relation, for example, patient factors and out-patient factors such as logistic and surgeon-related issues like sleep privation and fatigue. Moreover, it would be interesting to have epidemiological studies in Portugal (or in other countries, ideally multicountry), as well as studies about malpractice and associated complications. In conjunction, the study of these variables could contribute to create additional measures to reduce malpractice.

\section{Conclusions}

This study concluded that nighttime urgent surgery in patients with acute colorectal disease is not associated with greater postoperative medical-surgical morbidity risk when compared with daytime surgery. In fact, patients who underwent daytime colorectal surgery had longer hospital length of stay. Based in these results, the urgent procedures in patients with acute colorectal disease can be performed as urgent surgery, regardless of the time of the day.

\section{Compliance with ethical standards}

Conflict of interest The authors declare that they do not have conflicts of interest.

\section{References}

1. Kohn LT, Corrigan J, Donaldson MS (2000) To err is human: building a safer health system. National Academy Press, Washington, DC
2. Lee KT, Mun GH (2013) Is after-hours free-flap surgery associated with adverse outcomes? J Plast Reconstr Aesthet Surg 66:460-466

3. Bertram A, Hyam D, Hapangama N (2013) Out-of-hours maxillofacial trauma surgery: a risk factor for complications? Int J Oral Maxillofac Surg 42:214-217

4. Griner D, Adams A, Kotwall CA et al (2011) After-hours urgent and emergent surgery in elderly: outcomes and prognostic factors. Am Surg 77:1021-1024

5. Owens JA (2001) Sleep loss and fatigue in medical training. Curr Opin Pulm Med 7:411-418

6. Landrigan CP, Rothschild JM, Cronin JW et al (2004) Effect of reducing interns' work hours on serious medical errors in intensive care units. N Engl J Med 351:1838-1848

7. Eastbridge BJ, Hamilton EC, O'Keefe GE et al (2003) Effect of sleep deprivation on the performance of simulated laparoscopic surgical skill. Am J Surg 186:169-174

8. O'Loughlin E, Smithies WJ, Corcoran TB (2010) Out-of-hours surgery-a snapshot in time. Anaesth Intensive Care 38:1059-1063

9. Gray A (2000) United Kingdom national confidential enquiry into perioperative deaths. Minerva Anestesiol 66:288-292

10. Fechner G, Pezold C, Hauser S et al (2008) Kidney's nightshift, kidney's nightmare? Comparison of daylight and nighttime kidney transplantation: impact on complications and graft survival. Transplant Proc 40:1341-1344

11. Faiz O, Banerjee S, Tekkis P et al (2007) We still need to operate at night! World J Emerg Surg 2:29

12. Novack V, Jotkowitz A, Etzion O et al (2007) Does delay in surgery after hip fracture lead to worse outcomes? A multicenter survey. Int J Qual Health Care 19:170-176

13. George TJ, Arnaoutakis GJ, Merlo CA et al (2011) Association of operative time of day with outcomes after thoracic organ transplant. JAMA 305:2193-2199

14. Kienzl-Wagner K, Schneiderbauer S, Bösmüller C et al (2013) Nighttime procedures are not associated with adverse outcomes in kidney transplantation. Transpl Int 26:879-885

15. Rashid RH, Zubairi AJ, Slote MU et al (2013) Hip fracture surgery: does time of the day matter? A case-controlled study. Int J Surg 11(9):923-925

16. Dorotka R, Schoechtner H, Buchinger W (2003) Influence of nocturnal surgery on mortality and complications in patients with hip fractures. Unfallchirurg 106:287-293

17. Komen N, Dijk JW, Lalmahomed Z et al (2009) After-hours colorectal surgery: a risk factor for anastomotic leakage. Int J Color Dis 24:789-795

18. Eishove-Bolk J, Ellensen VS, Baatrup G (2010) Logistics and outcome in urgent and emergency colorectal surgery. Color Dis 12: 255-259

19. Biondo S, Pares D, Marti Rague J et al (2002) Emergency operations for nondiverticular perforation of the left colon. Am J Surg 183:256-260

20. Lee YM, Law WL, Chu KW et al (2001) Emergency surgery for obstructing colorectal cancers: a comparison between rightsided and left-sided lesions. J Am Coll Surg 192:719-725

21. Dindo D, Demartines N, Clavien PA (2004) Classification of surgical complications: a new proposal with evaluation in a cohort of 6336 patients and results of a survey. Ann Surg 240(2):205-213

22. Eddy R (2005) Sleep deprivation among physicians. Arch Surg 47: 176-180

23. McCormick F, Kadzielski J, Landrigan CP et al (2012) Surgeon fatigue: a prospective analysis of the incidence, risk, and intervals of predicted fatigue-related impairment in residents. Arch Surg 147: 430-435

24. Bonnet MH (2000) Sleep deprivation. In: Kryger MH, Roth T, Dement WC (eds) Principles and practice of sleep Medicin, 3rd edn. Saunders, Philadelphia, p 53 
25. Brandenberger J, Kahol K, Feinstein AJ et al (2010) Effects of duty hours and time of day on surgery resident proficiency. Am J Surg 200:814-818

26. ACSS. Novo Quadro Legal da Carreira Médica e Organização do Tempo de Trabalho Médico. [ACSS web site]. Apr 19, 2013. Available at: http://www.acss.min-saude.pt/Portals/0/Carreira\% 20m\%C3\%A9 dica FAQ-2013-04-19 final.pdf. Accessed Nov 08, 2013

27. Zorcolo L, Covotta L, Carlomagno N et al (2003) Toward lowering morbidity, mortality and stoma formation in emergency colorectal surgery: the role of specialization. Dis Colon Rectum 46:14611467

28. Biondo S, Kreisler E, Millan M et al (2010) Impact of surgical specialization on emergency colorectal surgery outcomes. Arch Surg 145:79-86

29. Biondo S, Parés D, Frago R et al (2004) Large bowel obstruction: predictive factors for postoperative mortality. Dis Colon Rectum 47:1889-1897 